\title{
Comparison of Recordings from Microelectrode Arrays and Single Electrodes in the Visual Cortex
}

\author{
Ryan C. Kelly, ${ }^{1,2 \star}$ Matthew A. Smith, ${ }^{1 \star}$ Jason M. Samonds, ${ }^{1}$ Adam Kohn, ${ }^{3}$ A. B. Bonds, ${ }^{4,5}$ J. Anthony Movshon, ${ }^{3}$ and \\ Tai Sing Lee Le $^{1,2}$ \\ ${ }^{1}$ Center for the Neural Basis of Cognition and ${ }^{2}$ Computer Science Department, Carnegie Mellon University, Pittsburgh, Pennsylvania $15213,{ }^{3}$ Center for \\ Neural Science, New York University, New York, New York 10003, and Departments of ${ }^{4}$ Electrical Engineering and Computer Science and ${ }^{5}$ Biomedical \\ Engineering, Vanderbilt University, Nashville, Tennessee 37235
}

Advances in microelectrode neural recording systems have made it possible to record extracellular activity from a large number of neurons simultaneously. A substantial body of work is associated with traditional single-electrode extracellular recording, and the robustness of the recording method has been proven experimentally. However, the recordings are limited to a small number of cells at a time, so much of the work has relied on compiling population statistics across many recording sessions. Multielectrode recording systems theoretically have some major advantages over this paradigm. They increase the yield of neurons per recording session, and analysis of pairwise correlation benefits greatly from simultaneously recording from a large number of neurons (the number of pairs is propor-

Received Nov. 10, 2006; revised Nov. 27, 2006; accepted Nov. 28, 2006.

This work was supported by a National Science Foundation (NSF) Integrative Graduate Education and Research Traineeship to R.C.K. (DGE0549352), National Eye Institute (NEI) National Research Service Award fellowship to M.A.S. (EY015958), National Institute of Mental Health Grant MH 64445 and NSF Grant CISE IIS 0413211 to T.S.L., and NEI grants to A.B.B. (EY014680) and J.A.M. (EY02017). Single-electrode cat data were collected by Ross Snider, cat array data were collected in part by Zhiyi Zhou and Melanie Bernard, and macaque single-electrode data were collected in part by Yasmine El-Shamayleh. We thank David Linn for technical assistance.

* R.C.K. and M.A.S. contributed equally to this work.

Correspondence should be addressed to Ryan C. Kelly, Center for the Neural Basis of Cognition, Carnegie Mellon University, 4400 Fifth Avenue, Mellon Institute, Room 115, Pittsburgh, PA 15213. E-mail: rkelly@cs.cmu.edu.

A. Kohn's present address: Department of Neuroscience, Albert Einstein College of Medicine, 1410 Pelham Parkway South, Bronx, NY 10461.

DOI:10.1523/JNEUROSCI.4906-06.2007

Copyright $\odot 2007$ Society for Neuroscience $\quad$ 0270-6474/07/270261-04\$15.00/0 tional to the square of the number of cells). The larger population also provides the possibility of examining higher-order (non-pairwise) interactions among neurons (Schneidman et al., 2006; Shlens et al., 2006). Finally, multielectrode systems have been developed that may be implanted and used for several months, which permits the study of learning in cell populations. Here we assess this experimental approach for anesthetized acute preparations and compare the quality of recordings to those provided by the traditional single-electrode method.

Here we focus on a specific microelectrode device, the Cyberkinetics "Utah" Array (Cyberkinetics Neurotechnology Systems, Foxborough, MA) (Fig. 1A). This device is a $10 \times 10$ grid of silicon microelectrodes ( $1 \mathrm{~mm}$ in length) spaced $400 \mu \mathrm{m}$ apart, covering $12.96 \mathrm{~mm}^{2}$. We analyzed recordings from single electrodes and the Utah array in macaques and cats. Previous reports on the quality (Nordhausen et al., 1996) and long-term stability (Suner et al., 2005) of array recordings have not made a quantitative, direct comparison with established singleelectrode recordings. In light of the increasing popularity of the array, we addressed this uncertainty by comparing waveforms recorded with the array to waveforms recorded with accepted singleelectrode techniques. We found that the array yields good recordings on a large number of electrodes, with qualities comparable to those from single-electrode re- cordings. On average, the recording quality is somewhat lower than that of single electrodes but, nonetheless, is sufficient for assessing tuning properties such as the spatiotemporal receptive field (STRF) and orientation tuning.

\section{Recording methods}

To assess the quality of microelectrode array recordings relative to standard singleelectrode techniques, we analyzed waveforms from single-electrode and microelectrode array recordings from anesthetized, paralyzed macaque monkeys (Macaca fascicularis) and cats (Felis domesticus). Anesthesia was maintained with sufentanil and propofol with nitrous oxide, respectively. Monkeys were paralyzed with vecuronium bromide, and cats were paralyzed with pancuronium bromide. The impedance of microelectrodes in the array ranged from 200 to $800 \mathrm{k} \Omega$ with an average of $400 \mathrm{k} \Omega$. For the macaque single-electrode recordings, we used quartz-platinum/tungsten microelectrodes (1.2-3 M $\Omega$ ) in a seven-channel Eckhorn microdrive (Thomas Recording, Giessen, Germany). For the cat singleelectrode recordings, we used tungsten in glass microelectrodes (0.5-2 M $\Omega$ ) made locally (Levick, 1972), in a Burleigh Inchworm microdrive (Burleigh Instruments, Victor, NY), driven by piezoelectric elements. All measures of impedance were made with a $1 \mathrm{kHz}$ sinusoidal current. Specific procedures for each of the following recording preparations have been re- 
ported previously: cat single electrode (Snider et al., 1998), cat array (Samonds et al., 2006), and monkey single electrode (Cavanaugh et al., 2002).

We used the following method to isolate waveforms generated by individual cells. For each electrode, waveform segments that exceeded a threshold (periodically adjusted using a multiple of the rms noise on each channel) were stored and sorted off-line with principal components analysis by waveform shape (Shoham et al., 2003). After this preliminary sort, we refined the output by hand with off-line time-amplitude window discrimination software for each electrode. All waveforms were sorted in this manner except for the V2 macaque data, which were sorted on-line with a dual timeamplitude window discriminator.

\section{Signal-to-noise ratio}

Using this procedure, we analyzed $58 \mathrm{~V} 1$ cells from single electrodes in cats, $38 \mathrm{~V} 2$ cells from single electrodes in macaques, $269 \mathrm{~V} 1$ cells from three microelectrode arrays in cats, and $301 \mathrm{~V} 1$ cells from three microelectrode arrays in macaques. In Figure $1 B$, we show waveforms from three neurons recorded with the microelectrode array in macaque V1. These examples span the quality range we typically observed with arrays. Given these isolated single units, we computed signal-to-noise ratios (SNRs) for collections of waveforms recorded in $1 \mathrm{~h}$ periods for each of the four preparations. The SNR is computed as the ratio of the amplitude of the average waveform to the SD of waveform noise (Nordhausen et al., 1996; Suner et al., 2005). That is, if each of $k$ waveforms has $n$ samples, then the collection of waveforms is as follows:

$$
W=\left[\begin{array}{c}
v^{1}\left(t_{1}\right), v^{1}\left(t_{2}\right), \ldots v^{1}\left(t_{n}\right) \\
\vdots \\
v^{k}\left(t_{1}\right), v^{k}\left(t_{2}\right), \ldots v^{k}\left(t_{n}\right)
\end{array}\right],
$$

with the mean waveform denoted as $\bar{W}$. The matrix of noise values (deviations from the mean) is thus as follows:

$$
\varepsilon=W-\left[\begin{array}{c}
\bar{W} \\
\vdots \\
\dot{\bar{W}}
\end{array}\right]
$$

where the $\mathrm{SD}_{\varepsilon}$ is the $\mathrm{SD}$ of the collection of all entries in $\varepsilon$. The SNR is now as follows:

$$
\mathrm{SNR}=\frac{\max (\bar{W})-\min (\bar{W})}{2 \times \mathrm{SD}_{\varepsilon}} .
$$

\section{Response properties}

Waveforms collected with the microelectrode arrays were similar in shape to wave-

A
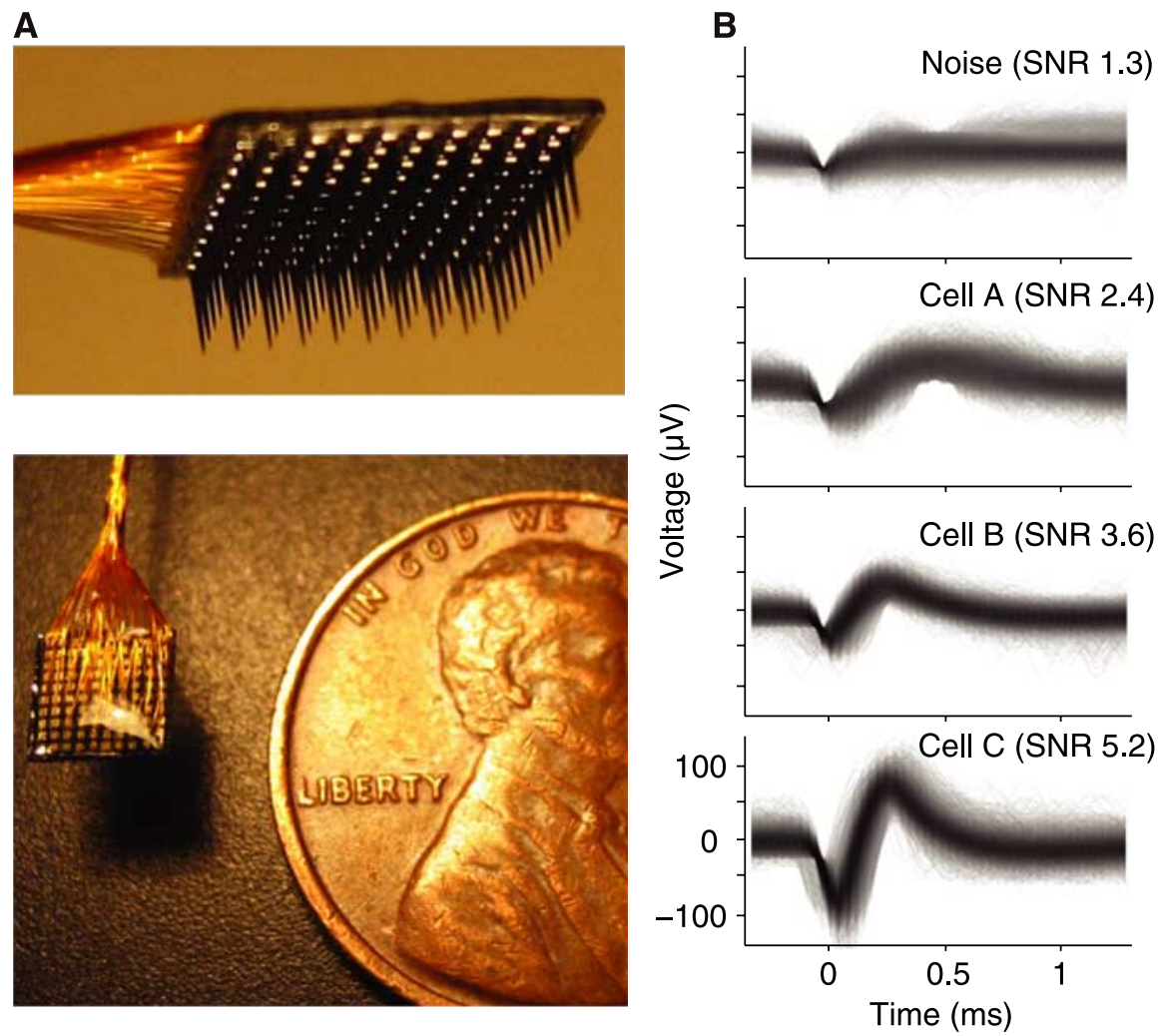

Figure 1. Cyberkinetics microelectrode array and example waveforms. $\boldsymbol{A}$, The array, closeup, and perspective with a penny. $\boldsymbol{B}$, Examples of sorted waveforms and SNRs from three representative channels and one channel of noise.

forms collected using single electrodes, and neuronal response properties were similar as well. To demonstrate this point, we analyzed neural responses to a variety of stimuli collected with macaque V1 array implants. Orientation tuning curves were derived from responses to drifting sinusoidal gratings (Fig. 2A). Most isolated cells showed clear orientation preference, and orderly shifts in orientation preference could be seen across the electrode positions in the array. In addition, we computed STRFs for cells responding to white-noise stimuli using spiketriggered averaging (Fig. $2 \mathrm{~B}$ ). STRFs were found for cells when a contiguous 30 pixel area of the spike-triggered average exceeded 3 SDs of the noise average. Of the cells isolated with this array, 60-65\% revealed STRFs. Overall, we found that tuning properties were similar to those reported previously for orientation tuning (Hubel and Wiesel, 1968; Ringach et al., 2002) and STRFs (Jones and Palmer, 1987).

We compared the distribution of SNRs across the different animals and methodologies (Fig. 3). On a population level, SNR values for the arrays tended to be somewhat lower than those from single- electrode recordings in both macaques and cats (see Fig. 3 legend for statistics). One reason for this is that the electrode depth of the array is fixed after implantation and cannot be adjusted to better isolate a cell, as is typically done in single-electrode recordings. Another possibility is that our array recordings were confined mostly to layers 2-3 with the rest in layer 4 (Jermakowicz et al., 2006), whereas our single-electrode data included cells sampled throughout the cortical depth. A direct comparison of cells recorded in the same layer might have yielded slightly different results, although there is no reason to suspect it would systematically bias the SNR values in either direction. The average SNRs from cat recordings were somewhat higher than from macaque data. This may be attributable to a true difference between species or merely related to variations in recording setups. Despite the disparity in distribution means, the cells in the different conditions span the same SNR range. That is, cells with the lowest SNR from arrays were still within the distribution from accepted single-electrode recordings using an on-line dual time-amplitude window discriminator. 
A
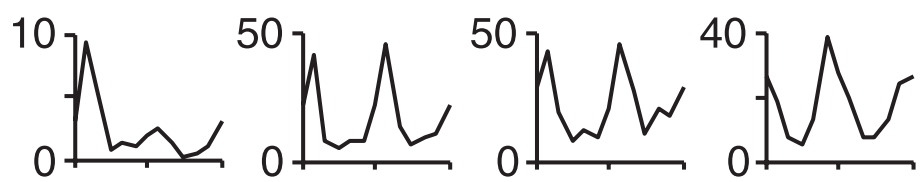

B
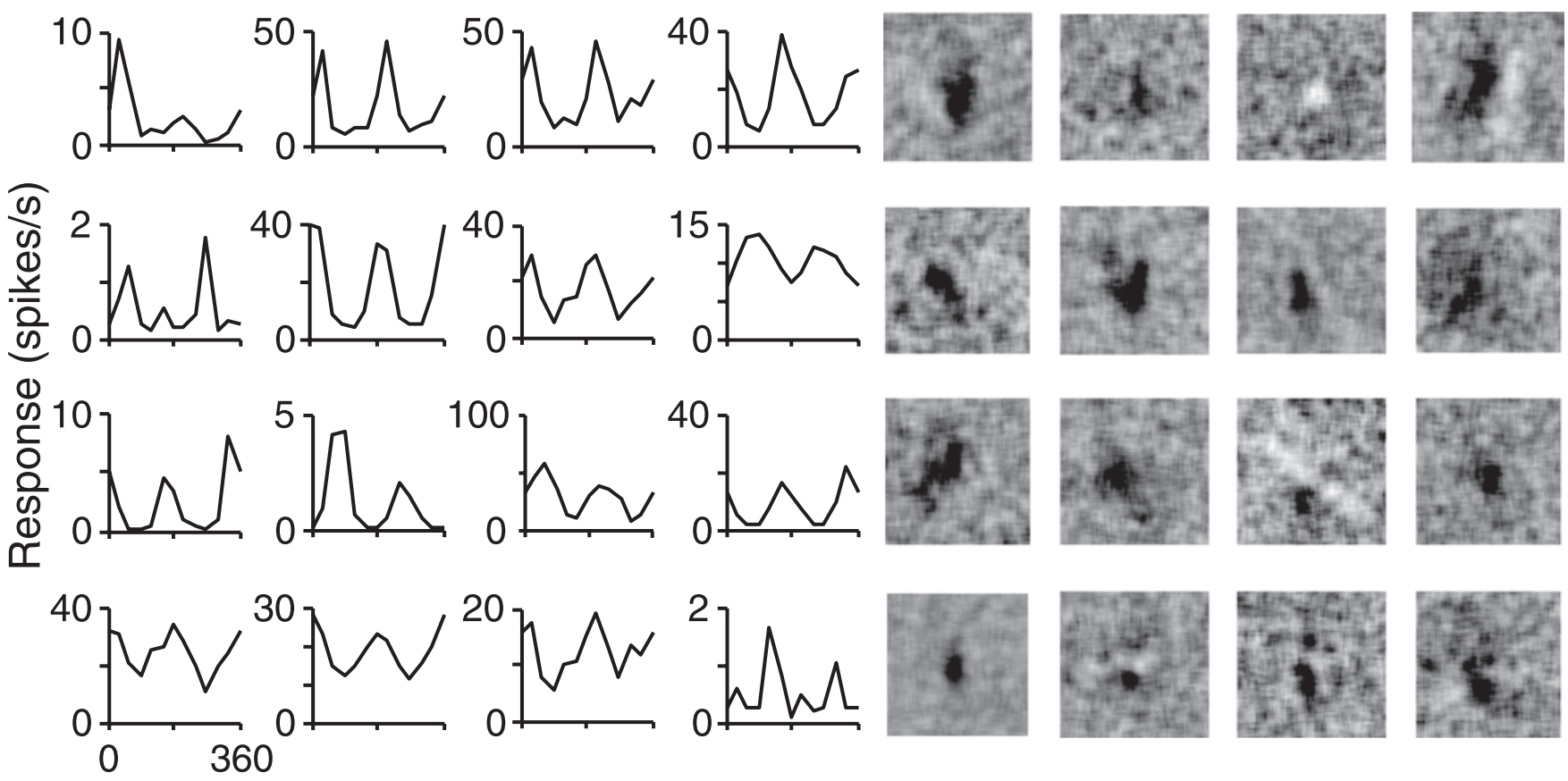

\section{Direction (degrees)}

Figure 2. Response properties. $A$, Orientation tuning curves for 16 example neurons in response to a sinusoidal grating drifting in 12 different directions. The grating was fixed at a spatial frequency of 1.3 cycles/degree, temporal frequency of $6.25 \mathrm{~Hz}$, size of $8^{\circ}$, and duration of $1.28 \mathrm{~s}$ with $1.5 \mathrm{~s}$ between stimuli. $\boldsymbol{B}$, STRFs generated with reverse correlation of responses to white-noise stimuli for the same 16 neurons.

\section{Stability of recordings}

One advantage of the acute preparation is the ability to record for many hours consecutively. We addressed the stability of recording across a $29 \mathrm{~h}$ period from one of our macaque array implants. Our sorting method was applied across the entire recording duration, and thus the quality of recordings from individual cells was tracked over time. Figure 4 shows the change in the SNR for all cells over the time period starting $2 \mathrm{~h}$ after array implantation. Most SNR values tend to remain relatively constant throughout recording, with a few fluctuating between high and low SNRs. One trend we observed was that cells had lower SNR values near implantation and improved over the course of recording (Pearson $r=0.27$; $p<$ $0.0001)$. Of 127 cells from this array, 88 $(69 \%)$ had a significant $(p<0.05)$ increase in SNR, whereas only 2 cells showed a significant decrease in SNR. Evolution of the mean SNR over time is shown at the top of Figure 4.

\section{Review of microelectrode array}

Use of the Cyberkinetics microelectrode array has some considerations relative to single-electrode techniques. It is currently manufactured in two fixed electrode lengths ( 1 or $1.5 \mathrm{~mm}$ ), and insertion
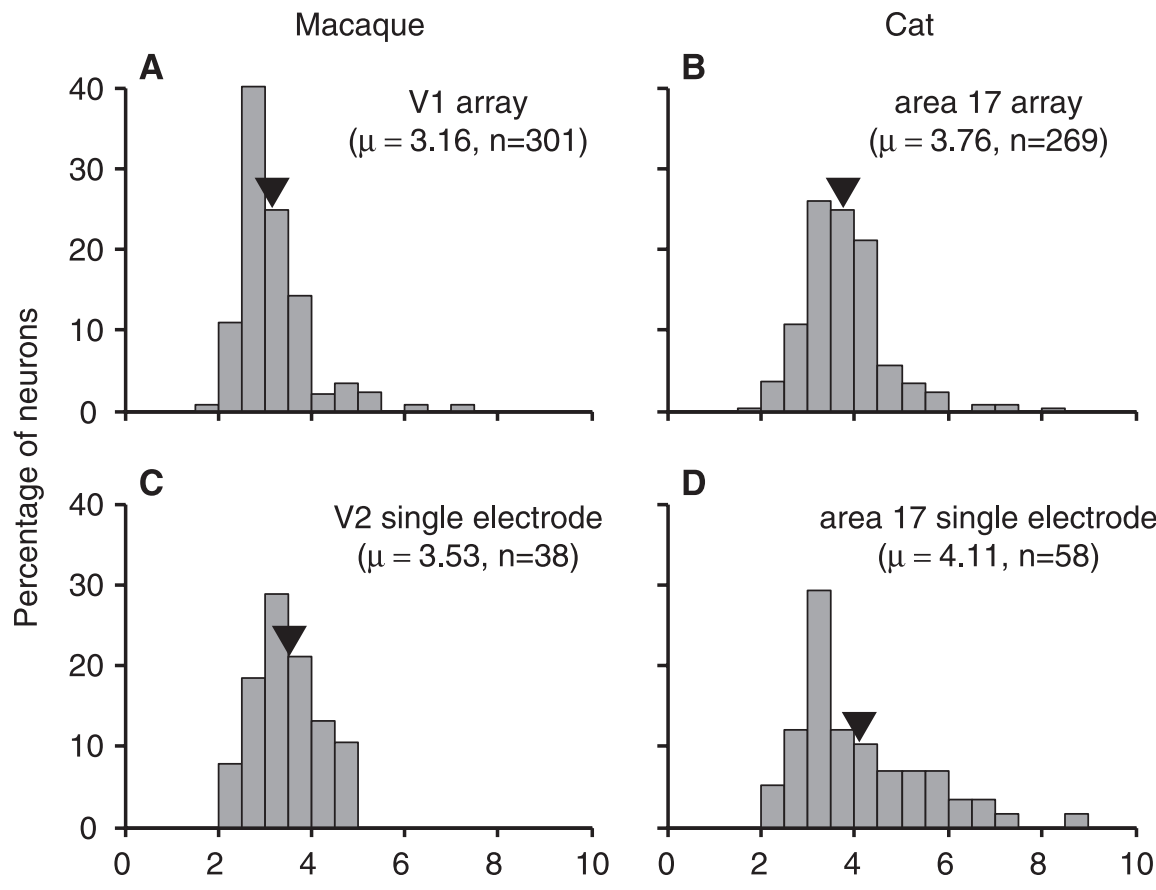

Signal to noise ratio (SNR)

Figure 3. SNRs in the four preparations. $\boldsymbol{A}, \boldsymbol{B}$, Macaque array SNR $(\boldsymbol{A})$ tended to be lower than single-electrode SNR ( $\boldsymbol{B}$; two-sample Kolmogorov-Smirnov goodness-of-fit hypothesis test, $p<0.0001)$. C, $\boldsymbol{D}$, Similarly, cat array SNR $(\boldsymbol{C}$ ) was lower than single-electrode SNR $(\boldsymbol{D} ; p=0.046)$. Finally, there was a trend for recordings in cats to have higher SNRs than recordings in the macaques for single electrodes $(p=0.10)$ and arrays $(p<0.0001)$. All SNR values were computed from the waveforms over $\sim 1$ $\mathrm{h}$ of recording time. 
depth is not adjustable after implantation. For our array recordings, we used a 1.0 $\mathrm{mm}$ array with a $0.6 \mathrm{~mm}$ pneumatic insertion (Rousche and Normann, 1992). This partial insertion resulted in electrode tips positioned mostly in superficial layers. An additional consideration is that the size and shape of the array prevents implantation in some locations accessible to single electrodes, such as within sulci. Also, because the array is flat and the cortical surface is curved, there is some chance that electrodes record from different layers. Finally, the implant procedure is somewhat more complicated than the preparation for single-electrode recordings (Rousche and Normann, 1992). However, we observed no significant damage or edema after implantation and even after removal of the array (potentially facilitated by partial insertion).

We found that the array produced SNRs similar to those of single-electrode recordings, both when the same spikesorting method was used and when spikes were sorted with standard on-line techniques. Although the ranges of SNR distributions were similar, single-electrode recordings had higher SNR values on average. This may be resulting from the higher impedances of single electrodes but is also likely to be strongly influenced by the fixed electrode depth of the array. The stability of recording may be influenced by the fact that single-electrode setups are mounted externally, whereas the array is allowed to float with the motions of the cortex resulting from heartbeat and respiration. Our recordings from arrays had orientation tuning and STRFs in most cells. These cells were relatively stable throughout the day after implantation, with no signs of degradation after $30 \mathrm{~h}$ (indeed, the signals tended to improve over time). A typical array implantation yielded 100 distinct candidate waveforms split approximately evenly between single-unit and multiunit activity. Aside from increasing the amount of single-unit data, arrays produce a high yield of neuronal pairs, providing a viable foundation for the study of higher-order correlation properties.

\section{References}

Cavanaugh JR, Bair W, Movshon JA (2002) Nature and interaction of signals from the recep-
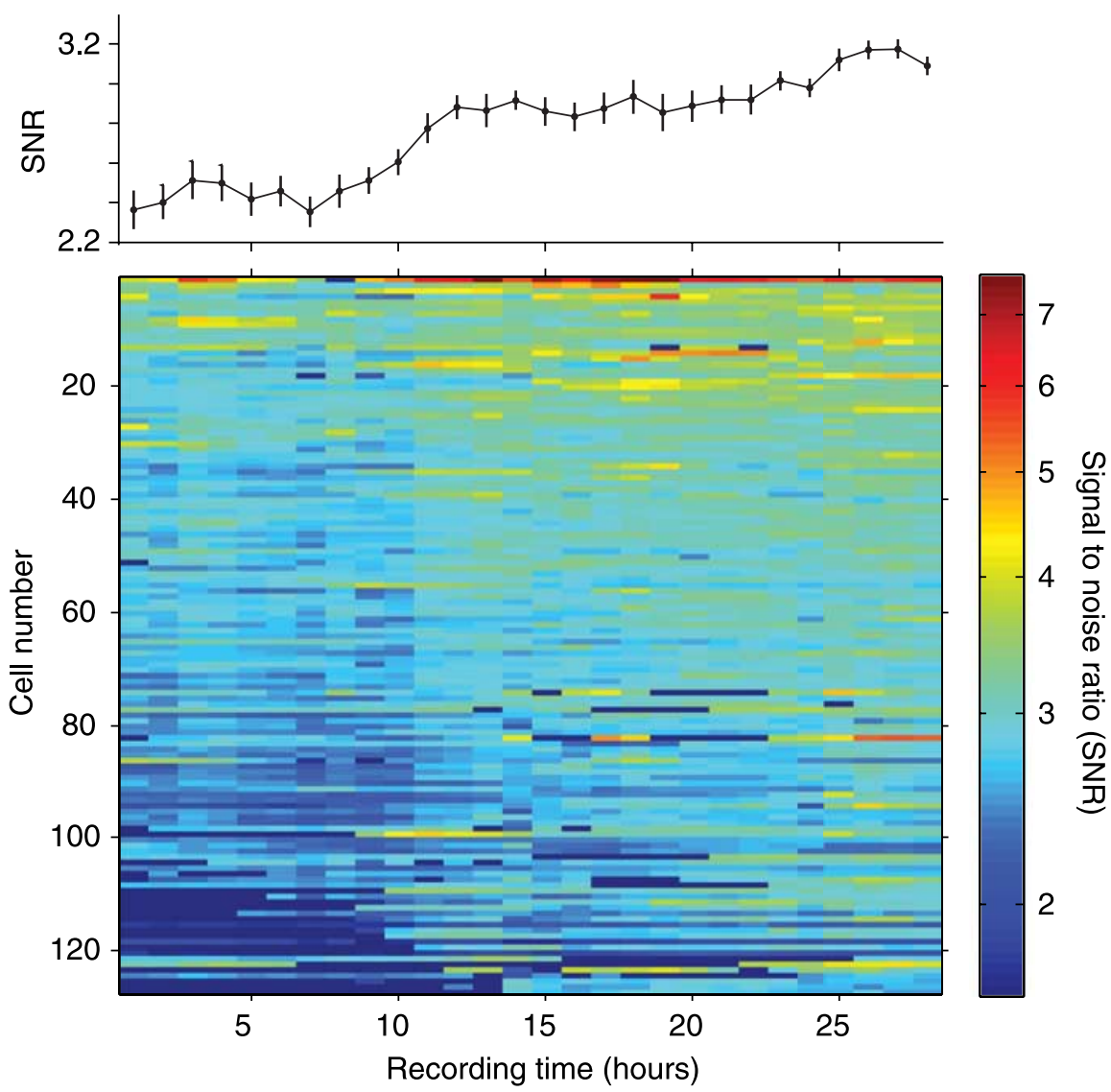

Figure 4. Stability of SNR. Here we show the $\log$ (SNR) values for a continuous $29 \mathrm{~h}$ recording session from one array implant. The cells here are sorted in decreasing order by average SNR value across time. The plot above shows the average SNR across all cells for each hour (error bars are \pm 1 SEM).

tive field center and surround in macaque V1 neurons. J Neurophysiol 88:2530-2546.

Hubel D, Wiesel T (1968) Receptive fields and functional architecture of monkey striate cortex. J Physiol (Lond) 195:215-243.

Jermakowicz WJ, Chen X, Khaytin I, Zhou Z, Bernard M, Bonds AB, Casagrande VA (2006) Does spike synchrony provide a better code of stimulus angle than average firing rate? J Vis 6:66a.

Jones JP, Palmer LA (1987) The two-dimensional spatial structure of simple receptive fields in cat striate cortex. J Neurophysiol 58:1187-1211.

Levick W (1972) Another tungsten microelectrode. Med Biol Eng 10:510-515.

Nordhausen CT, Maynard EM, Normann RA (1996) Single unit recording capabilities of a 100 microelectrode array. Brain Res 726:129-140.

Ringach DL, Shapley RM, Hawken MJ (2002) Orientation selectivity in macaque V1: diversity and laminar dependence. $\mathrm{J}$ Neurosci 22:5639-5651.

Rousche PJ, Normann RA (1992) A method for pneumatically inserting an array of penetrating electrodes into cortical tissue. Ann Biomed Eng 20:413-422.
Samonds JM, Zhou Z, Bernard MR, Bonds AB (2006) Synchronous activity in cat visual cortex encodes collinear and cocircular contours. J Neurophysiol 95:2602-2616.

Schneidman E, Berry MJ, Segev R, Bialek W (2006) Weak pairwise correlations imply strongly correlated network states in a neural population. Nature 440:1007-1012.

Shlens J, Field GD, Gauthier JL, Grivich MI, Petrusca D, Sher A, Litke AM, Chichilnisky EJ (2006) The structure of multi-neuron firing patterns in primate retina. J Neurosci 26:8254-8266.

Shoham S, Fellows MR, Normann RA (2003) Robust, automatic spike sorting using mixtures of multivariate t-distributions. J Neurosci Methods 127:111-122.

Snider RK, Kabara JF, Roig BR, Bonds AB (1998) Burst firing and modulation of functional connectivity in cat striate cortex. J Neurophysiol 80:730-744.

Suner S, Fellows MR, Vargas-Irwin C, Nakata GK, Donoghue JP (2005) Reliability of signals from a chronically implanted, silicon-based electrode array in non-human primate primary motor cortex. IEEE Trans Neural Syst Rehabil Eng 13:524-541. 